\title{
Generation of neural progenitor cells by chemical cocktails and hypoxia
}

\author{
Lin Cheng ${ }^{1}$, Wenxiang $\mathrm{Hu}^{1}$, Binlong Qiu ${ }^{1}$, Jian Zhao ${ }^{1}$, Yongchun $\mathrm{Yu}^{2}$, Wuqiang Guan ${ }^{2}$, Min Wang ${ }^{2}$, \\ Wuzhou Yang ${ }^{2}$, Gang Pei ${ }^{1,3}$ \\ ${ }^{I}$ State Key Laboratory of Cell Biology, Institute of Biochemistry and Cell Biology, Shanghai Institutes for Biological Sciences, Chi- \\ nese Academy of Sciences, 320 Yueyang Road, Shanghai 200031, China; ${ }^{2}$ Institute of Neurobiology, Institutes of Brain Science and \\ State Key Laboratory of Medical Neurobiology, Fudan University, Shanghai 200032, China; ${ }^{3}$ School of Life Science and Technol- \\ ogy, Tongji University, Shanghai 200092, China
}

Cell Research (2015) 25:645-646. doi:10.1038/cr.2015.55; published online on 5 May 2015

Correction to: Cell Research (2014) 24:665-679. doi:10.1038/cr.2014.32; published online on 18 March 2014

In the initial published version of this article, an error was made during the assembly of Figure 6C. Figure 6C displays representative images of morphology and immunostaining analysis of passage 16 ciNPCs (ciNPC p16) induced from TTFs, which shows that ciNPC p16 have typical NPC morphology and express NPC marker genes Nestin, Sox2 and Pax6. After the initial publishing of this article, it has been brought to our attention that the images of monolayer and Nestin/Sox2/DAPI staining in previous Figure 6C look similar to certain panels in Figure 5B. Upon careful examination of the original data, we realized that these two images in previous Figure $6 \mathrm{C}$ were indeed erroneously assembled. Specifically, the monolayer image in previous Figure 6C partially overlapped with the monolayer image of NLS-induced ciNPC p13 in Figure 5B, while the Nestin/Sox2/DAPI image in previous Figure 6C almost completely overlapped with the Nestin/Sox2/DAPI image of TLT-induced ciNPC p13 in Figure 5B. All images in Figure 5B are correct, while the wrong images shown in previous Figure $6 \mathrm{C}$ were acutally photos taken from the samples of Figure 5B under different but overlapping field views. We are sorry that these wrong images were inadvertently assembled into previous Figure 6C. The corrected Figure 6C and its legend are provided below. This correction does not affect the description of the results in the paper or the conclusions of our study. We wish to thank the reader who brought the error to our attention. We also deeply apologize for any inconvenience that may have been caused by our error.
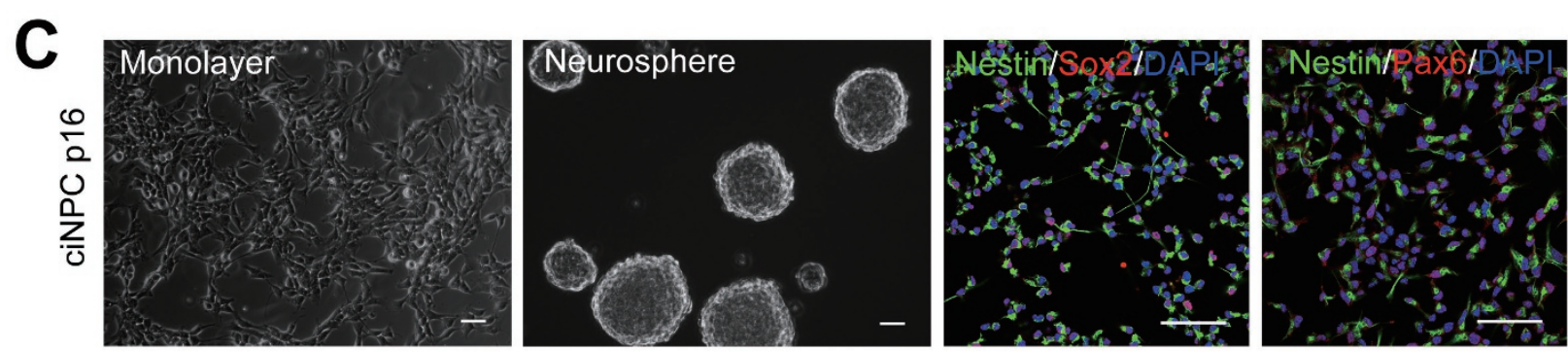

Figure 6 (C) Morphology and expression analysis of Nestin, Sox2 and Pax6 of ciNPCs at passage 16 induced from TTFs. 
There is also an error in the Supplementary information: the link to Figure S9 was previously wrongly pointed to the pdf file of Figure S3. This error was introduced during the web production of the paper. The corrected link is now installed in the online version of the paper.
The microarray data reported in the paper can be download via the following link (GEO accession number: GSE68311).

http://www.ncbi.nlm.nih.gov/geo/query/acc. cgi? acc $=$ GSE68311 